\title{
ON THE RELATION BETWEEN THE POSTERIOR COLUMNS OF THE SPINAL CORD AND THE EXCITO-MOTOR AREA OF THE CORTEX, WITH ESPECIAL REFERENCE TO PROF. SCHIFF'S VIEWS ON THE SUBJECT.
}

\author{
BY VICTOR HORSLEY, B.8., F.R.O.S., \\ Assistant Surgeon to University College Hospital; Surgeon to the National \\ Hoopital for the Paralysed and Epileptio, Queen's Square; Brovon \\ Profeseor of Pathology to the University of London.
}

THE problem which most attracts the attention of all classes of neurologists, whether clinical, experimental, or anatomical, is the identification of function with certain spots in the brain, and discovery of the channels of communication which unite the central nervous system with a harmonions whole. It is the discussion of the relation between the afferent paths in the spinal cord and the motor cortex which concerns us in this paper, renewed attention. having been lately drawn to the subject by the recently published experiments of Prof. Schiff. Briefly we may state the case, with a slight preamble, as follows. Every one admits the necessity of regarding the whole central nervous system as being built up on the plan of (1) an afferent channel leading to (2) a sensory centre, which is in close communication with (3) a motor centre from which issues (4) an efferent channel.

Now the bare existence of these constructive factors being granted, it is only their anatomical arrangement which is hotly disputed. There may be said to be three chief views on this subject.

A. The "Separatist" View.-This view places the sensory (perceptive) centres in a distinct, though neighbouring, part of the cortex from the motor centres whioh are situated in the excitable area of the same. The posterior columns or afferent channels, therefore, are in direct communication with 
the former of these centres, and only reach the latter (the motor) indirectly by the commissural connections between the centres.

B. The "Fusion" View.-A favourite mode of regarding the subject on the Continent appears to be one in which the sensory (at least that for tactile sensibility) centre is fused with the motor centre. This view, it is to be supposed, carries with it the assumption, that the posterior columns of the spinal cord are in direct communication with the motor cortex.

C. Prof. Schiff's View.-Prof. Schiff states that he has traced (physiologically) the afferent fibres (of the posterior columns of the cord) directly to the excitable portion of the cortex, which he therefore regards as being the sensory perceptive centre for tactile sensibility. From this sensory perceptive area he believes that fibres go down to the motor centres, which are situated he knows not where.

To recapitulate; it is clear that, according to the first view, (2) and (3) are situated in different parts of the cortex, (3) being identical with the excitable area. In the second view, (2) and (3) are fused together in the excitable area; while finally, according to Schiff, (2) is identical with the excitable area, and (3) is somewhere else.

This much being clear, it is now my intention to detail at length Prof. Schiff's mode of reasoning and experimentation, which has led him to the above conclusion. I shall then point out the fallacies in the same, and finally describe fully the experiments I made last autumn to investigate this point. We owe the unusual opportunity of learning much of Prof. Schiff's work to the scientific zeal and energy of Dr. W. R. Huggard, who communicated to the 'Lancet' of Ang. 1, 1885, a paper describing the above-mentioned rork, and entitled, "Professor Schiff's Experiments on the Excitable Area of the Cerebral Cortex." In that paper he states pretty fully the way in which Schiff looks at the subject, and his method of investigating the same. Before entering on the practical side of the matter in dispute, I shall criticise the line of reasoning and the points of explanatory meaning, which occur in Dr. Huggard's report.

Such examinatory criticism must necessarily be somewhat disjointed, as the tale which is told in the above-mentioned 
paper is, in the nature of things, a general essay on the work of the distinguished Genevese Professor. Possibly, however, very little difficulty will be met with in picking up the general thread of the argument. We may note at the outset an enunciation (p. 8 of separately published copy of Dr. Huggard's paper $^{1}$ ) of the function of the excitable area of the cortex which is intended to be descriptive of the action of a sensory perceptive centre, but which, as a matter of general understanding, is, more properly speaking, a perfect explanation of the function of a motor centre. The passage runs thus: "The grey matter of the excitable area transmutes. sensibility into a liberating (probably voluntary) current." The usual interpretation which is put on such a term as "sensibility" is the appreciation by a sensory perceptive centre of message conveyed thereto by an afferent channel, for consciousness is connoted. Hence Prof. Schiff's real position is that the excitable area is a portion of brain which transmutes the energy received from a sensory perceptive centre into a motor impulse ("voluntary current") and that is one which all upholders of the first or separatist view (A. before described) will cordially agree to.

It seems to me very necessary to dwell upon these apparently trivial differences in modes of expression, for it is just ambiguity in the meaning of terms which of itself creates diversity of opinion where very often agreement really exists. To pursue this point a little further, we find that Prof. Schiff (and following him Dr. Huggard too) is disposed to consider that certain apparently paretic (I say apparently, merely for the sake of argument, as I shall show directly the condition is one of real paralysis) conditions are capable of being referred to as ataxia. Perhaps it will be better to illustrate Prof. Schiff's position by the statement of the experimental result on which he bases this opinion. He has found that removal of the sigmoid gyrus, on the one hand, and the effects (after four or five days have elapsed) of cutting the posterior column of the cord on the other, are both productive of similar symptoms, viz: paresis (voluntary paralysis, really),

\footnotetext{
1 Wherever paging of Dr. Huggard's paper is mentioned hereafter, it is to be anderstood as belonging to the Separate publication.
} 
and that this apparent paresis disappears when the animal runs about on a rough surface. The condition following these operations he calls ataxia.

The adoption of this term to express the condition which results from destruction of the sigmoid gyrus might perhaps be justified, if the exactly similar symptom was eroked by section of the posterior columns of the cord only. Prof. Schiff, of course, considers this latter point is established by his experiments; but I shall show directly that (to use Dr. Huggard's words) "they do not warrant the inference," and in fact that "an essential factor has been overlooked." 'To proceed with the examination of this so-called ataxic condition, it will be well worth while to describe in greater detail the effects of removal of the sigmoid gyrus in the dog, and the more especially since the hiatuses in Prof. Schiff's description, or perhaps I should more correctly say Dr. Huggard's report, happen to include the omission of some of the most characteristic features. Thus the position of the paresed limbs is very constant and striking. The animal stands resting mainly on the limbs of the opposite side, and with the toes of the paralysed side doubled up, so that the dog walks really on the dorsum of the manus and pes respectively. Precisely the same condition sometimes follows section of the posterior columns of the cord, for the simple reason (see p. 5) that the pyramidal tracts frequently degenerate at the seat of operation, and further, exactly the same condition follows section of the principal nerve-trunks in the limb itself. Next, if thorough examination be made of such an animal, it will be found that there is complete paralysis of voluntary movement, the animal is unable to purposely extend the toes and wrist, \&c. It should really be scarcely necessary at the present time to point out again the enormous difference in the proportion of cortical representation of the peripheral muscular segments in the dog as compared with other animals, but from internal evidence in the above-mentioned report it does not appear that this fact is fully appreciated yet. The amount of differentiation exhibited by the various so-called centres is extremely small in the dog. Even the extensor muscles of the paw and digits, the representation of which 
may be considered as the acme of cortical erolution in the dog, can very rarely be called into solitary action by stimulating the cortex even with minimal currents. And just as local differentiation in the extreme point of cortical evolution only crudely exists, so also the extent of the excitable area for the limb movements is extremely small compared with the rest of the brain, hence it is clear that in such an animal the very primitive phenomena of equilibration and progression are most -unlikely to be disturbed by removal of the mechanism of those volitionary impulses which occasionally mollify them. To sum up this point, it is clear that whatever part of the volitional motor tract be injured, whether it be the nerves in the limb (provided, of course, all the motor nerves.in the limb ure not cut, but only those supplying the two main distal segments), the pyramidal tracts in the cord, the internal capsule or the cortex itself, the result is the same as might have been expected. It only remains now to take note of the two points on which most stress is laid by Prof. Schiff, namely, first his assertion, that removal of the excitable area in the dog is followed by loss of tactile sensibility on the opposite side; and secondly, his "crowning evidence" (pp. 6, 7), which consists of the following observation: "In dogs whose posterior columns were cut in the cervical region, if, after the lapse of about four days, the socalled motor region is laid bare, it is found on both sides to be no longer excitable. If one posterior column only is cut, the opposite side of the brain only is inexcitable. If the posterior columns or posterior roots (?) are cut in the lumbar region, irritation of the cortex still produces movements of the anterior limbs, but not of the posterior."

Now as regards the first of these two points, viz. the asserted disappearance of tactile sensibility after removal of the opposite sigmoid gyrus, I cannot speak from personal observation on dogs, and therefore simply refer the reader to Professor Ferrier's 'Functions of the Brain,' pp. 216-217, where this question and that of ataxia too, is sufficiently discussed. We may therefore put aside the first point and take up the second, viz. his attempted demonstration of the direct extension of the posterior columns of the cord to the sigmoid gyrus 
of the opposite side. His method of experimentation, so far as can be made out from the necessarily short description given in the before-mentioned paper, consists simply of exposing and dividing one posterior column, say the left; then preserving the animal for a period of not less than five days; then exposing the opposite sigmoid gyrus and stimulating it with the ordinary induced current, and observing the absence of the usual movements of the left limbs. The section of the posterior column was usually made opposite, about the fourth cervical nerve, and the motor centres for the fore-limbs of the same side tested as described.

The whole gist of this experiment, as ingeniously designed by Prof. Schiff, lies in the provision of the interval of five days between the section of the cord and the testing of the opposite motor gyrus. For he considered that the consequential ascending degeneration of the posterior column would, in that space of time, reach the opposite cortex and cause degeneration of the grey matter there, which grey matter, we have already seen, he believes to be the perceptive centre of tactile sensibility.

My criticism of this experiment is that it does not justify the conclusions drawn from it by Prof. Schiff until microscopic examination has shown that (1) there is such degeneration of the sigmoid gyrus, and (2) that there are no further changes in the spinal cord than the lesion and degeneration of the posterior column. Feeling the extreme importance of deciding the question thus raised by Prof. Schiff, I determined to repeat his experiment, directing my special attention to these two points; and it will be seen from the results I publish below, that in the first place no such degeneration of the sigmoid gyrus can be demonstrated; and in the second place that Prof. Schiff"s "crowning evidence" in support of his position is entirely nullified by the fact, that the section of the posterior column of the cord sets up local degenerative changes in the motor tracts of the cord. ${ }^{1}$

I shall now proceed to expand this latter statement, by

1 Fnglish neurologists at any rate will be interested to learn (vide 'Ĺancet of the 20th of February, 1886) that Prof. Schiff is about to combat the general opinion that the crossed lateral tract is motor. 
detailing the way in which I have repeated Prof. Schiff's experiment and the results I have obtained.

The animal, a dog, being very deeply ${ }^{1}$ narcotised with ether, an incision was made directly over the fourth cervical spine, which being removed, a trephine hole was made through the laminæ of the fourth cervical vèrtebra. (As a rule the fourth was chosen, but in some of the experiments the spinal canal was opened through the fifth.) The dura mater was then incised by a little transverse cut, and the posterior surface of the spinal cord exposed. After the flow of cerebro. spinal fluid had stopped, the left posterior column of the cord was carefully divided by a small puncturing incision made with a sharp-pointed tenotomy knife. The wound was then closed completely, full antiseptic precautions being taken. On this latter point it is necessary to digress a little. $I$ have performed seven experiments; in two of these the antiseptics failed, owing to the fact, that the animals were so deeply narcotised as to cause cessation of respiration, \&c. This necessitated artificial respiration, \&c., the spray being discontinued and the windows opened, hence the contamination of the wound. In the remaining five the hole in the spine and dura mater was found by me to be completely plugged, with a dense fibrous plaque of cicatricial tissue, and the soft parts completely united; in two instances the skin being bulged by a collection beneath it of wound serum. In the two instances in which the antiseptics failed, the animals of course suffered from septic meningitis; the subdural space in one being distended with pus, while in the other the arachnoid as far down as the middle of the dorsal region was raised from the cord by pent-up sero-pus. The pus of the wound in each of these two cases was fetid, having a sickly odour, and containing numerous putrefactive bacilli. The advent of this septic condition had only this influence on the experiment, viz. that it made Prof. Schiff's result come ont very markedly, the reason of which is obvious.

To return to the method of experimentation. ${ }^{2}$ With the

1 On account of the exquisite sensibility of the dura mater.

2 I desire here to express my indebtedness to my friend Dr. Beevor for kindly assisting me in the record of several of the following observations. 
two exceptions given above, the animals did not appear inconvenienced, except by the motor paresis, \&c. During the five days' interval between the completion of the abovedescribed operation and the exploration with induced current of the two cortices, careful examination was made to find whether there was any motor or sensory paresis to be discovered in the left fore-limb. The testing of the former was of course designed so as to make it clear whether the disturbance of voluntary movement was such as to cause incapability of performing purposive acts. As regards sensation, the testing of tactile sensibility was performed in three different ways. In the first place the skin was touched lightly and painlessly by means of a pin; in the second place, I used the spring clip referred to by Prof. Schiff; lastly, I devised as a specially sensitive test the injection of water on to the part (the nerve reaction of which may have been hampered), the sensibility of which was in question. At the outset, I think it very desirable that it should clearly be understood by those who have to estimate the value of original research in this direction, how very difficult it is to correctly gauge the appreciation by one of the lower animals, and especially a dog, of tactile impressions. It is not scientific to draw hard-and-fast lines of distinction between the sensory appreciation of touch, pain, temperature, \&c., although of course there is actual difference between the perception of these various physical conditions; in short, since we are usually dealing with combinations of any two or more stimuli - stimulation, as a rule, is compounded of several factors-it would seem only natural that the paths conveying such stimuli are not likely to be very highly differentiated. This nondifferentiation is of course simple enough to understand as an outcome of such confusion of sensory function, but it must not be left out of mind when we come to consider the appreciation of such different sensory stimuli as those I have already described. I will now enumerate the facts which I observed in my own experiments bearing on this and the foregoing point.

As I have found the affection of sensation to be coupled with the affection of movement (i.e. voluntary paralysis, Schiff's 
ataxia) in a very suggestive way they may, with advantage, be considered together; and as the motor paresis is by far the easier to recognise, and to explain in accordance with the wellunderstood function of certain traots in the spinal cord, I shall take that first.

Paresis.-Of the whole number seven of experiments, the question as to whether the condition which I have shown to be perfectly typical of paralysis of voluntary command of the distal segments of the fore-limb existed or not, could only be determined in six, as the first was complicated too much by septic meningitis. Of the remaining six, this paresis was present in three and absent in the rest, although in every case the complete division of the posterior oolumn hàd been perfectly effected.

Here, then, is a very definite result, which of itself would be quite sufficient to negative the view that the paresis was a form of ataxia; for though the channels of afferent impressions (according to Schiff) had been destroyed in three cases, there was no "ataxy" produced at all. It is clear therefore that the paresis must be due to damage done to a motor tract lying in proximity to the area of section. Now the two well-known divisions of the pyramidal tract, the direct and crossed portions, are both in proximity to the area divided; for while the former, situate in the anterior column, is only separated by the commissures from the posterior column, the crossed lateral tract is still nearer, being only divided from the latter by the slender posterior cornu of grey matter. In every case one or both of these divisions of tha motor tract was degenerated (as will be explained directly), in very varying degree. The cases in which no paresis was observable were those in which the degeneration was very slightly marked; while in those instances where it was severe, the paresis was very definite. This fact of "cortical" i.e. voluntary paralysis following a localised patch of acute (vide infra) degeneration in the pyramidal tract, will not of course be surprising to any one (save those who question the motor function of the pyramidal tract, vide note, p. 47); but connected with this observation of the formation of a local block in the motor tract, as a consequence of the division of the posterior column, is the question of the 
degree and causation of the tactile angesthesia referred to by Prof. Schiff.

The exact relations, i.e. corpuscle and ganglion-cell connections of the fibres of the posterior columns of the cord, are not yet accurately known, all, in fact, that we do know of their function being derived from experiments (artificial and natural) on the effects which follow their damage. Certain it is from such data that if one posterior column be divided, ascending degeneration can be traced from the point injured as high as the bulb. Whether all the fibres in the column are thus disorganised as far as the top of the spinal cord, is another matter, and one which $I$ cannot enter on now. Granting, then, that the fibres of the posterior column are afferent as to the nature of their function, I consider that the results of two of the experiments (viz. III. and IV.) in which there was no appearance of disturbance of tactile sensibility, although the posterior column was most completely divided, and the ascending degeneration very. well marked, throw grave doubt on the idea that the posterior columns convey afferent tactile impressions. In further confirmation of this idea, which, resting as it does on two experiments only, is certainly open to the charge of being too slightly founded, I wish to note fully the instances, also two in number (Experiments II. and VI.), where this loss of tactile sensibility was well marked. In these cases (II. and VI.) there was undoubtedly tactile anæsthesia; but there was in addition very definite motor paresis, and of course, as explained above, distinct degeneration in the lateral column. Now this couple of cases, II. and VI., presents the exact converse of the condition seen in III. and IV., where the affection of the motor tract was very slight indeed. Hence we are brought to this juncture: Is the loss of tactile sensibility due to degeneration attacking the direct cerebellar tract in the most posterior portion of the lateral column, or to a similar damage of other afferent fibres in the lateral column? It is, of course, quite superfluous to discuss the existence and function of the direct cerebellar tract, but it is worth while to pause for a moment, and to remember that the exceedingly careful experiments of Miescher demonstrated (by accurate 
measurement of the variations of blood-pressure) that afferent fibres of so-called vaso-motor influence travelled up to the encephalon in the lateral column of the spinal cord. Degeneration, we have just seen, was present in the lateral columns in my experiment when tactile anæsthesia was noted, and again this latter symptom was absent in the cases where the degeneration was scarcely visible. So here we have the crucial test. I cannot help feeling, therefore, that the question as to the path in the spinal cord of the impressions which arouse tactile sensibility must remain undecided. ${ }^{1}$

To sum up. After the above described operation on the spinal cord, paresis and tactile anxsthesia were only present in those instances where degeneration had attacked the anterolateral columns.

I will now take up the last division of my subject, and present the results of stimulation of the cortex of the side opposite to the lesion of the posterior column in the spinal cord.

As however the whole seeming abnormal deficiency of function in the excitable area is most obviously due to the degeneration I have referred to, situated in the pyramidal tract opposite the seat of operation and not, as Prof. Schiff would have us believe, in the excitable area, I think it will be best to describe the state of the spinal cord in each of $\mathrm{my}$ experiments.

The spinal cord operated on as above was examined in the the following way. After the cortex on each side had been thoroughly explored (vide infra) the cord was rapidly exposed (the animal being deeply etherised), and then being still living, the contrast between the dead white of the degenerated ascending posterior column which had been divided, as compared with the almost semi-translucent appearance of the normal one, was very striking.

The cord was then remored and hardened in a 2 per cent. solution of bichromate of ammonia, to which $\frac{1}{3}$ volume of

1 Not wishing to stretch my point beyond what some might consider justifiable limits, I have purposely called the asceuding fibres, described by Miescher as of "so-called raso-motor influence," but it will be remembered that they were channels of conduction for affurent impressious producerl by stimulating the great sciatic nerve. 
methylated spirit was added at the end of a fortnight. Two portions of the cord were then taken, viz. (1) the seat of operation with the neighbouring $6 \mathrm{~mm}$. above and below the point cut, and (2) a small piece opposite the 1st or 2nd cervical nerve; these were soaked in water, then in gum, and finally cut after freezing. One half of the sections were then stained with aniline blue-black, the other with logwood, - and finally mounted in oil of cloves and Canada balsam. This method of preparing the sections, although rapid, had the disadvantage of allowing the more friable grey matter of the posterior cornu, forming, as it necessarily does, the outer boundary of the wound in the spinal-cord to partly break awny in some cases during the preparation of some of the sections, as is represented in the accompanying outlines of the various sections. (See pp. 56,57.) The exact delimitation of the lesion to the posterior column was very obvious during the cutting of the preparations in the microtome. The condition of the wound in each case was closely similar, so that one description will serve for all. It consisted simply of a microscopic slit extending from the surface of the cord to the posterior commissure, and containing a little wound-serum and debris of damaged nerve-fibre, the mouth of the wound being always closed by young but firm connective tissue. The appearanco of the sides of the wound-cavity, although so extremely small, showed clearly that there was some degree of tension in it; tension therefore was present in the interior of the cord, a factor that seems to me of some pathological importance in the correct estimation of the effects of such wounds of the cord. The chief point, however, which calls for particular notice in this method of research is the degeneration in some of the parts of the cord contiguous to the seat of operation, and of which a great deal has already been said. We may conveniently discuss this important point under the headings Nature, Locality, and Causation. The part which it plays in producing Prof. Schiff's result I have already referred to at full length, therefore now only wish to draw attention to the details of its existence.

(1.) Nature.-Prof. Schiff, it will be remembered, insisted on an interval of a few days being allowed to elapse, vOL. $1 \mathrm{x}$. 
in order, as he said, that the degeneration might reach the cortex. Now how far the ascending degeneration of the posterior columns gets in four or five days, I do not know, not having traced the course of the degenerated fibres; but this much is perfectly evident, that it does not arrive at, and cause the destruction of, the ganglion-corpuscles in the sigmoid gyrus, as Prof. Schiff would have us believe, for, microscopically, these corpuscles show no degenerative changes. But it is plain that this interval allows of the degeneration in the motor tracts of the spinal cord about to be described. Now the nature of this degeneration is very simple, consisting, as it does, merely in the swelling of axis-cylinders and myelin sheaths, so as to form the well-known and classical picture of the histological changes produced in nerve-fibres by acute "inflammatory" mischief within that space of time. In the accompanying drawings, where the shading has been marked darkly, there it is to be understood that the degeneration was very complete, that is to say, that all the nerve-fibres in the degenerated area were destroyed. To prevent misapprehension on this point, it will be well to draw attention to the fact that this complete destruction was only to be found in the direct cerebellar tract, the crossed lateral tract, and the direct pyramidal tract of the same side as the wound in the cord. I mention this particularly, since in Nos. 2 and 5 it will be noticed that the degeneration is necessarily represented diagrammatically as being spread over the whole lateral column, because degenerated fibres could be detected throughout that half of the section, although complete destruction was limited to the tracts above noted. It is scarcely necessary to describe the histological details at length, since, as has been stated above, they presented the ordinary appearances. It will make, however, this account more complete if I briefly state the chief facts :-

Changes in-

(A.) Nerve Fibres.-Axis Cylinders. The axis cylinders of the fibres involved in the degenerative process remain unaltered at first, since they can be detected in the centre of the swollen myelin (vide infra). 
Soon, however, they atrophy, and in the majority of instances the space (dilated) in the neuroglia formerly occupied by the fibre is simply filled with a deeply staining myelin globule. The continuity of the fibre is thus completely interrupted.-Myelin. The myelin exhibits the first effects of the damaging process. It swells, and at first is but feebly stained by aniline blue-black; then it swells more, so as to fill the space in the neuroglia, and it stains nearly black. Finally, it appears to shrink again, and is absorbed or, as a rule, simply drops out in the preparation of the section.

(B.) Neuroglia.-The changes in the neuroglia, as might be expected, are not very marked, owing to the shortness of the period of time during which they might develop. In fact, as regards the fine supporting trabeculae of connective tissue running with the capillaries, the only notable point about them is that they take the staining more deeply on the degenerated areas. The fine meshwork between them appears unaltered, save by the distension of the fibre spaces before referred to. As is noted in the description of each of the spinal cords at the seat of operation, the grey cornua in the neighbourhood of the wound are in most cases infiltrated to a greater or less extent with leucocytes, and there is also under these circumstances a distinct appearance of odema.

(c.) Blood-Vessels.-The degenerated area in every case was very anæmic, and in one instance, No. 4, this was extremely marked, while in the right half of the cord the vessels were full of blood. The importance of this fact will be discussed directly. (vide Causation, infra.)

(2.) Locality.-The exact locality of the degeneration is shown in the accompanying sketches of the cords, vide pp. 56, 57 , but attention may be drawn with advantage to one or two cardinal facts determining its distribution. 
Thus at the first glance it will be seen that the mischief is almost confined to the side of the cord operated upon. Next, that it is most marked in the closely adjoining posterior third

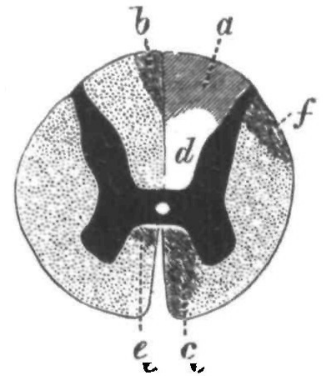

Fig. 1.-Central Canal distended, and contained exudation and blood.' The left posterior cornu was infiltrated with leucocytes for half its breadth, and in some sections through its whole thickness.

Septio Case.-Pia mater was thickly infiltrated with leucocytes (pus). Schiff's result, marked.

$a=$ Left posterior column destroyed, t.e. track of knife. $b=$ Small portion of right p. $c$. degenerated. $c=\bar{W}$ hole direct pyramidal tract of left anterlor column v(ry greatly degenerated. $d=$ Cavity of wound. $c=$ Slight degeneration of direct pyramidal tract of right anterior $\infty$ lumn. $f=$ legeneration of ascending direct cerebellar tract.

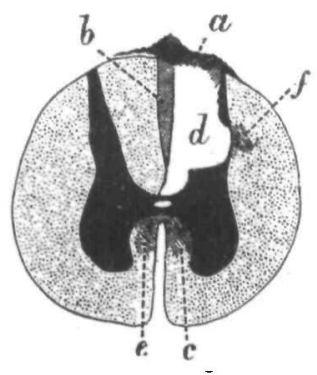

Fig. 3.-Central Canal contains blood clot.

Aseptio Case.-Schifrs result, very slight.

$a=$ Scar tlesue plugging wound. $b=$ Romalns of left posterior column destroyed. $c=$ Degeneration, very glight in left anterior column. $d \rightleftharpoons$ Wound cavity. $e=$ Degeneration of right anterfor column, slight. $f=$ Sligbt adema (?) of crossed lateral tract.

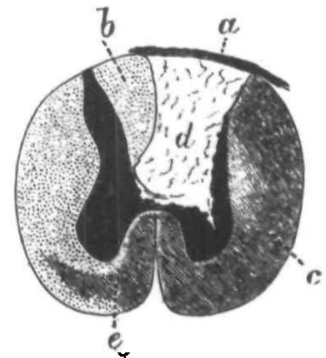

Fig. 2.-Central Canal was normal. It is not represented in the figure, which is a copy of the microscopical preparation. The left cornua were much jnfiltrated with leucocytes, and here and there putches of extravasated blood.

Aseptic Case.-Schiff's result, marked.

$a=8 \mathrm{car}$ tissue closing wound. $b=$ Right posterior column (2rds.). $c=$ Area of degeneraton on left olde involving whole antero-lateral column, $d=$ Wound cavity full of detris. (Note. The damage done appears much larger, as already explained, ow.ng to the friab:lity of the " in. flamed " grey substance.) $c=$ Megeneration in the direct pyramidal (ract of the right anterior column.

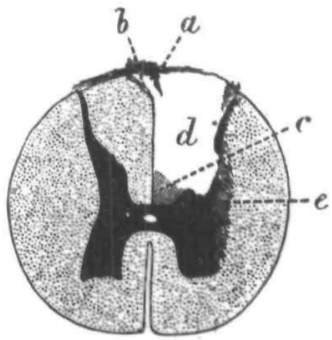

Fig. 4.-Central Canal moderately distended with exudation. The left pasterior cornu was inflitrated wilh leucocytes at the notch represented in the drawing.

Aseptic Case. Bchiff's result, extremely olight.

$a=$ Scar tissue closing wound. $b=$ Small angle of right posterfor colnmn cut away. $c=$ Small patch of degenerated left posterior column. $d=$ Cavity of wound. $e=$ Small vertical ratch of degeneration Just outside cormu of grey matter. 
of the lateral column. Thirdly, that the next place of greatest frequency is the direct pyramidal tract in the vertical line of the wound. The explanation of the greater liability of these areas to be affected will be discussed directly, vide Causation,

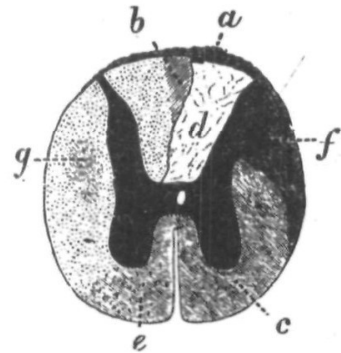

'ig. 5.-Central Canal normal. Whole cord, but especially left grey cornu, is infiltrated slightly with louoceytes. Septic Case. - Cord oodematous. Soliff's result, excessively marked, seo p. 6.

$a=$ Scar Issacue and pia mater. $b=$ Damaged portion of right postertor column. $c=$ Degenerated left antero-lateral column. $d=$ Cavity of wound filled with debris of left posterior column. $e=$ Degenerated direct pyramldal tract in right anterior column. $f=$ Degeneration of crossed lateral tract In left half of cord. $g=$ Very slight degeneration in right crossed lateral iract.

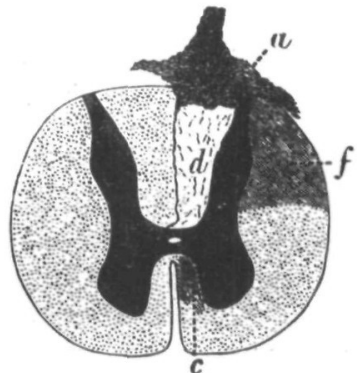

Fig. 6.-Central Canal normal. Loft cornu infiltrated with leucocytes. Aseptic Case. Sohiff's result, marked.

$a=$ Scar tissue. $d=$ Cavity of wound filled wilh debris. $c=$ Degeneration in direct pyramidal tract of left anterior column, $f=$ Crossed lateral tract and cerebellar tract much degenerated to exact limit ahown.

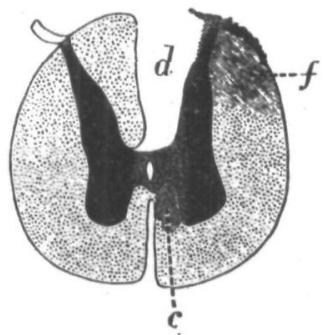

Fig. 7.-Central Canal normal.

Aseptic Case. Schiff's result, very slight.

$d=$ Wound cavity. $c=$ Degeneration in left anterior colnmn. (Tblo was extremely limited.) $f=$ Well-marked degeneration of the cerebellar tract, extending but slightly into left crossed lateral tract.

infra; but I must now state that the degeneration is only marked exactly opposite the wound in the cord, and only extends a millimetre above and perhaps two millimetres below that point. This narrow limitation of the degeneration 
proves it to be an entirely local effect of the operation. I mention this, because it might possibly be imagined by some that ascending degeneration might, as Schiff believes, go up to the sigmoid gyrus, destroy the corpuscles there, and then come down the pyramidal tract. In each case I have examined the cord microscopically, not only just above and below the seat of operation, but also opposite the 1st and 2 nd cervical nerves, and in no instance was any degeneration observable in the motor tracts at the summit of the cord. We may now discuss the causation of this degeneration.

(3.) Causation.-In the first place it is to be remembered that, with the exception of Nos. I. and V., each experiment was absolutely aseptic, and therefore we may set aside sepsis as a cause of the degeneration. From a consideration of the facts that the degeneration was most marked in proportion to

1. The amount of distension of the wound-cavity, in the cord and this meaus also the density and size of the cicatricial plug closing it;

2. The amount of anæmia and damage to blood-vessels; it is clear that we have suggested to us two ways in which this overlooked source of fallacy may be caused. These two possible ways are, tension in the cord, and interference with the blood-supply. In the first place, tension will obviously tell most heavily on the neighbouring crossed lateral tract. In the second place, the whole vascular supply to the left posterior quadrant of the cord will be interfered with by the operation, and further than that, a certain amount of thrombosis may occur which would reach the median artery, and so cut off the supply to the direct pyramidal tract. It would not be profitable to go over again the evidence offered in the foregoing pages, proving that both of these conditions follow the division of the posterior column, but it is worth while to note, that, as expressed also in the Table on p. 62, where these conditions were only slightly present the results as described by Schiff were very feebly marked, and vice versî.

Having now seen clearly that a little localised patch of 
acute degeneration (plus tension in the cord) attacks invariably, though in very different degree, the motor tracts in the spinal cord on the same side as a wound of the posterior column, and that such a patch and such pressure necessarily form a block, offering greater or less resistance to the passage of motor impulse from the excitable cortex, we may turn to review the results obtained by stimulating the cortex in these experiments, as directed by Prof. Schiff. It will be remembered that in every case the left posterior column was the one divided. In the first two experiments I first exposed and tested the right excitable area, and then subsequently (i.e. ten or fifteen minutes later) the left. In the remaining five experiments I first removed the bone from the dura mater over both the motor cortices, and then raised the dura mater so that the exposure of the two sides was practically simultaneous, while in testing, the electrodes were first tried on one side and then on the other. This slight difference in the mode of treating the cortex on the two sides, as might be expected, made no difference in the results obtained. As will be seen from the subjoined Table, the amount of movement of the fore-limb of the left side as compared with that of the right side, when the right and left sigmoid gyri were respectively stimulated, varied inversely as the amount of degeneration visible in the motor tract of the cord opposite the seat of operation. Consequently it is erroneous to describe the diminution of movement as being due to a decrease in the excitability of the sigmoid gyrus, when it is really owing to the fact, that of course the swollen axis-cylinders in the degenerated lateral columns are incapable of transmitting the energy discharged from the irritated cortex. This experimental result is an interesting illustration of the clinical observation of paralysis from acute "inflammatory" conditions in the spinal cord. It will not be necessary to take up space with the minute details of all the cases experimented upon, but I may give short notes of Experiment V. as illustrating, first, the most extreme condition of Prof. Schiff's result; secondly, the fact that (as I have mentioned before) this result was obtained to such a very marked degree because, owing to the wound becoming septic, the degeneration was more 
extensive; and finally, the general method followed. The following is an abstract of the details of this experiment.

\section{Experiment V.-Dog, Aduit Mongrec Terrier.}

Sept. 11th, 1885.-Left posterior column divided (according to method described above). Animal asphyxiated by ether, but recovered by artificial respiration.

12th.-Dog apparently well.

13th.-Marked paralysis of left fore-paw. Lies habitually on left side.

In attempting to walk, frequently falls to left side (hemiplegic).

14th.-During previous night, symptoms of spinal meningitis noted.

At 2.20 P.M. both fore-legs rigid, general rigidity of trunk, \&c., with the other classical symptoms of meningitis. Dog immediately anæsthetised with ether.

2.45 P.M.-Both sigmoid gyri exposed within two minutes, and then stimulated us above described.

(I may here take this opportunity of stating, that in this series of experiments the apparatus of stimulation were an ordinary Du Bois Reymond'induction coil, one Daniell cell [in one case, two cells were used] and usual platinum electrodes. The divisions indicating the distance of the secondary from the primary coil are given in centimetres; the strongest current, therefore, being at zero.)

$$
\begin{aligned}
& \text { Corrent. Right Sig. Gyrus. Left Sig. Gyrus. } \\
& 10 \mathrm{~cm} . \text {. . . Nil. . Nil. } \\
& 8 \mathrm{~cm} . \text {. . . Nil. . . Nil. } \\
& 6 \mathrm{~cm} \text {. . Flickerings in left . Fibrillar twitchings in right } \\
& \text { trapezius. trapczius und protrusion of } \\
& \text { limb with extension of fore- } \\
& \text { paw. } \\
& 5 \frac{1}{2} \mathrm{om} \text {. . . Ditto. . . Ditto, stronger. } \\
& \text { Interval, i.e. rest of } 5 \text { minutes. } \\
& 5 \mathrm{~cm} \text {. . . Ditto, . . Ditto, and sprend to head, and } \\
& \text { no spread. right lower limb. }
\end{aligned}
$$

"The difference was so extremely marked as not to need further investigation."

In fact a superficial examination would have led one to 
state that the right sigmoid gyrus, as far as the centres for the limb-and trunk-muscles respectively were concerned, was inexcitable. As a matter of fact however, in this, as in all other experiments, the right cortex was perfectly excitable, as evidenced by the active movements of the facial and cervical muscles as low as the distribution of the fourth cervical nerve. I have already described the condition of the spinal cord in this case (see page 57), in which description it will be remembered that the degeneration of the motor tract was most marked. This case will thus serve as a typical illustration of the mode in which I believe Prof. Schiff"s result is always produced. Of course it was impossible in this instance that the nerve energy should reach the left limb muscles.

The rest of the facts concerning the production of Schiff"s result, viz. the diminution in the usual amount of muscular movement evoked by stimulation of the opposite sigmoid gyrus, so invariably illustrate (vide Table, p. 62) the fact alluded to before, viz. the variation of degree of loss of power, being proportionate to the damage of the lateral columns, that but little addition is necessary to the details just enumerated. There are, however, one or two points which are of considerable interest. In the first place, in those cases where Schiff's result was scarcely obtained at all it was only obvious when powerful currents were employed. Moderate, but very adequate stimuli called forth the usual movements on both sides, if a little different in force, but with such $a$ stimulus as a current at $5 \mathrm{~cm}$. this difference in violence of the movement became very apparent. The second point is this, that in three instances there was noted very definite clonic tremor (post-ethereal) of a rate of ten to twelve per second, which occurred in the left forelimb first before invading the others. This fact seems to me worthy of note, since it appears to indicate that ordinary clonic tremor, so familiar clinically, is, as I suggested in my Brown Lectures, 1885, not the simple question of a rhythmical alternation of contraction of opposing groups of muscles, but that it (ankle-clonus, for example) is really the rhythmical contraction of a given muscle (e.g. the gastrocnemius) in response to a rhythmical discharge of the spinal motor corpuscles governing 
that muscle, such corpuscles having been thrown into discharging activity by the sudden violent afferent impulse which is generated (according to the usual clinical method) by forcibly stretching that muscle, e.g. by flexion of the ankle. 'The pathological irritability of the motor corpuscles in my experiments obviously is set up by the changes I have described in the descending pyramidal tracts, an experimental condition comparable to the pathological one of sclerosis.

Conclusion.-The inference to be drawn from the above experimental examination of the question whether Schiff's method shows the posterior columns to ascend directly to the motor or excitable cortex must, I think, be that such direct connection is not proven. The investigation (the expenses of which were defrayed by a grant of $5 l$. from the British Medical Association) has elicited the fact, not, so far as I know, published before, that tension and anæmia in the cord will bring about marked local degeneration in the motor tract especially.

Table showing the relation between the Degenfration in the Anterolateral Columss and the Effects which were supposed to be due to Lesion of the Posterion Coluan, this being in every case completely divided.

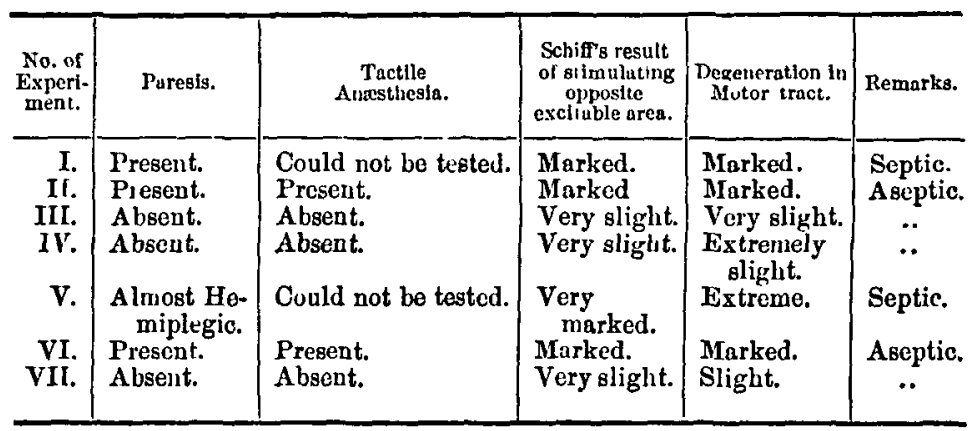

Note.-When Schiff's result of want of movement following stimulation of the right sigmoid gyrus as compared with that foilowing excitation of the left is set duwn in varying degree in the 4th column, it is to be remembered that the terms in which it is described are exact, since they are founded on the experimental data which are the mensurements of the strength of the current in contimetres of the distance of the secondary from the priwary coil; sce p. 60 . 